\title{
INCREASED INTESTINAL VEGF EXPRESSION AND MUCOSAL VASCULARIZATION IN PATIENTS WITH SPONDYLARTHROPATHY.
}

\author{
P. Hindryckx (1), G. Serry (1), D. Laukens (1), H. Peeters (1), M. De Vos (1) / [1] Gent
}

BACKGROUND: Chronic intestinal inflammation observed in about a quarter of patients with spondylarthropathy (SpA) is linked to Crohn's disease (CD). Increased expression of Vascular Endothelial Growth Factor (VEGF) and intestinal mucosal angiogenesis has been described in Crohn's disease, in correlation with disease activity.

STUDY AIM: To investigate VEGF expression and mucosal angiogenesis in SpA patients with or without subclinical gut inflammation.

METHODS: Sections of paraffin-embedded ileal and colonic biopsies from healthy controls (HC) (negative control, number of biopsies $(\mathrm{N})=20$ ), Crohn patients (positive control, $\mathrm{N}=20$ ), SpA patients without gut inflammation $(\mathrm{N}=30)$ and SpA patients with subclinical gut inflammation ( $\mathrm{N}=28)$ were immunostained for VEGF and for CD31 and blindly scored by computerized morphometric analysis using specialized software.

RESULTS: Both intestinal VEGF activity and mucosal vascular density (MVD) were higher in SpA patients versus healthy controls, independent of the presence of chronic gut inflammation (HC vs SpA patients without gut inflammation: $\mathrm{P}<0,05$ and $\mathrm{P}<0,01$ respectively for VEGF and MVD; HC vs SpA patients with chronic gut inflammation: $\mathrm{P}<0,001$ both for VEGF and MVD). Nevertheless, presence of subclinical chronic gut inflammation was associated with much stronger intestinal VEGF expression and mucosal vascularization, resembling the pattern of Crohn's disease (SpA patients with gut inflammation vs SpA patients without gut inflammation: $\mathrm{P}<0,001$ for VEGF; $\mathrm{P}<0,01$ for MVD). Unlike in Crohn patients however, the high VEGF activity and MVD observed in SpA patients with chronic gut inflammation was not clearly dependent on the presence of active inflammation in the biopsy (epithelial infiltration of polymorphonuclear cells, ulcerations, granulomas).

CONCLUSION: A pro-angiogenic intestinal phenotype is observed in SpA patients which, unlike in Crohn's disease, is not clearly dependent of active gut inflammation. 